\title{
Mental Pain in Suicidal Individuals of the New Millennium
}

Maurizio Pompili

Prof. Maurizio Pompili, M.D., is Full Professor and Chair of Psychiatry as part of the Faculty of Medicine and Psychology at Sapienza University in Rome, Italy, where he received his M.D. degree and completed his training in psychiatry (both summa cum laude). He is also the director of University Psychiatric Clinic, the director of Suicide Prevention Center at Sant'Andrea Hospital in Rome. Prof. Maurizio Pompili was also part of the Community at McLean Hospital - Harvard Medical School, the USA, where he received a fellowship in Psychiatry. He is the recipient of American Association of Suicidology's 2008 Shneidman Award.

Abstract. Among the constructs used to describe the wish to die, a simple but extraordinary model has proved, for its straightforwardness, to be useful in describing the suicidal mind. Edwin Shneidman first posited that the suicidal individual experiences unbearable psychological pain (psychache) or suffering and that suicide might be, at least in part, an attempt to escape from this suffering. Shneidman considered psychache to be the main ingredient of suicide. According to this model, suicide is an escape from intolerable suffering, emphasizing that suicide is not as a movement toward death but rather as an escape from intolerable emotion, unendurable or unacceptable anguish. Experiencing negative emotions, with an internal dialogue making the flow of consciousness painful and leading the individual to the ultimate conclusion, may be related to the fact that, if tormented individuals could somehow stop consciousness and still live, they would opt for that solution. Suicide occurs when that individual deems the psychache to be unbearable. The author will describe new results on a multi-centre observational study involving patients who were administered a battery of tests assessing suicidal ideation and behaviour, mental pain, depression and childhood maltreatment. Results point to the fact that suicide attempters (compared to non-attempters) had higher odds of reporting worse psychological pain and suicidal intent with/without a specific plan. They also had higher odds of having a personality disorder and major depression. Contrary to our hypotheses, higher degrees of childhood trauma were not associated with more severe mental pain scores. The author will also cover the role of mental pain during the time of the pandemic. 